\title{
The Fusion Circular RNA F-circEA1 Promotes Non- small Cell Lung Carcinoma Progression through ALK Downstream Signaling
}

yinping Huo ( $\sim$ billinda@126.com )

Nanjing Jinling Hospital: East Region Military Command General Hospital https://orcid.org/00000001-6937-0964

\section{Tangfeng LV}

Nanjing Jinling Hospital: East Region Military Command General Hospital

Mingxiang Ye

Nanjing Jinling Hospital: East Region Military Command General Hospital

Suhua Zhu

Nanjing Jinling Hospital: East Region Military Command General Hospital

Jiaxin Liu

Nanjing Jinling Hospital: East Region Military Command General Hospital

Hongbing Liu

Nanjing Jinling Hospital: East Region Military Command General Hospital

Yong Song

Nanjing Jinling Hospital: East Region Military Command General Hospital

\section{Research}

Keywords: Circular RNA, F-circEA1, EML4-ALK, NSCLC, ALK

Posted Date: April 5th, 2021

DOI: https://doi.org/10.21203/rs.3.rs-368666/v1

License: (a) (1) This work is licensed under a Creative Commons Attribution 4.0 International License. Read Full License 


\section{Abstract}

Background Circular RNA has a stable closed structure, which plays an important role in the occurrence and development of tumors. However, there are no reports on the relationship between fusion circular RNA and EML4-ALK variant 1, or their underlying molecular mechanisms in non-small cell lung carcinoma (NSCLC).

Methods To test F-circEA1 in NCl-H3122 cells (carrying the EML4-ALK variant 1 gene) by RT-PCR, FISH and Sanger sequencing; To determine cell proliferation with a CCK-8 assay. Transwell experiments were used to detect cell migration and invasion. Cell cycle stage and apoptosis were detected by flow cytometry. The sensitivity of cells to crizotinib was tested using a CCK-8 assay. RT-PCR and western blots were used to measure the expression of EML4-ALK1 and downstream signaling factors associated with ALK. Subcutaneously transplanted tumors were used in nude mice to determine the effect of F-circEA1 on tumor formation and the expression of EML4-ALK1 by immunohistochemistry. Statistical analyses were carried by GraphPad Prism 8.0 and differences between groups were compared using Student's t test. Difference with $p$ value $<0.05$ is statistically significant.

Results F-circEA1 was present both in the cytoplasm and nucleus of $\mathrm{NCl}-\mathrm{H} 3122$ cells. F-circEA1 was contributed to cell proliferation, metastasis, invasion. F-circEA1 induced cell arrest, promoted cell apoptosis and improved drug sensitivity to crizotinib. After knockdown with F-circEA1, subcutaneously transplanted tumors in nude mice grew slowly, the expression of EML4-ALK1 in tumor tissues decreased significantly. The mRNA and protein levels of EML4-ALK1 decreased after knockdown of F-circEA1 but increased after its overexpression. The protein expression of downstream ALK signaling factors increased after overexpression of F-circEA1, but not at the mRNA level.

Conclusions We have confirmed a potential carcinogenenic and therapeutic role for F-circEA1 in NSCLC. Our experimental results may provide a new biomarker, treatment method, and prognostic monitoring index for use in the clinic.

\section{Background}

Non-small cell lung cancer (NSCLC) is still one of the leading causes of cancer-related death worldwide. Although great progress has been made in treatment regimens including immunotherapy and targeted therapy, the clinical prognosis of NSCLC patients has been little improved due to the recurrence and metastasis of lung cancer are still difficult to control [1]. Approximately $85 \%$ of lung cancer patients present with non-small cell lung cancer (NSCLC) [2] and patients positive for the EML4-ALK fusion gene represent a subpopulation of these. The EML4-ALK fusion gene is formed by the translocation of EML4 and ALK genes on chromosome 2[3]. The cleaved terminal ends of EML4 are different and therefore, this makes the fusion site different in ALK, resulting in several EML4-ALK isomers. The most common EML4ALK fusion variants are 1 and 3 , which together account for about $60 \%$ of EML4-ALK positive lung cancer 
cases [4]. Unfortunately, existing treatments for NSCLC, such as chemotherapy, radiotherapy, and molecular targeted drugs, have limited efficacy.

In recent years, studies have found that circular RNA plays an important role in tumor progression, and cancers with chromosomal translocations can produce associated fusion circRNA, this F-circRNA can affect tumor cell viability, promote cell transformation and have tumor-promoting properties [5]. Recently it has been found that F-circSR derived from the SLC34A2-ROS1 fusion gene, can promote cell migration in NSCLC [6].

Currently, EML4-ALK variant 1 associated F-circRNA and its potential function have not been reported in NSCLC. Our study, using FISH and sanger sequencing, has found that EML4-ALK variant 1 produced a fused circular RNA termed F-circEA1, which affected tumor proliferation, migration, invasion, apoptosis and other biological functions and promotes tumor progression and has drug resistance to crizotinib. Furthermore, it had interesting effects on the expression of the parental gene EML4-ALK1 and the downstream signaling pathway of ALK. Utilizing in vivo studies and the use of a lentivirus to interfere with F-circEA1, we have confirmed that interference with F-circEA1 can inhibit tumor growth and inhibit the expression of the parental oncogene EML4-ALK1. Therefore, our experimental results may provide a new biomarker, treatment method, and prognostic monitoring index for use in the clinic.

\section{Materials And Methods}

\section{Cell lines and cell culture}

Three NSCLC cell lines, A549 and SPCA1(no EML4-ALK1 gene), H3122 (EML4-ALK1 positive cells), as well as HBE (human bronchial epithelial cells) were purchased from the Cell Bank of the Type Culture Collection Committee of the Chinese Academy of Sciences and had been passed the STR cell identification in 2020. All cell lines have been tested for mycoplasma contamination before the experiment. These cells were cultured in RPMI 1640 medium (Gibco-BRL, USA). The cell culture medium contained 10\% fetal bovine serum (FBS), (Gibco-BRL, USA) and 1\% penicillin/streptomycin (Gibco-BRL, USA). Cells were cultured in a humidified incubator containing $5 \% \mathrm{CO}_{2}$, and at $37^{\circ} \mathrm{C}$.

\section{Plasmid construction and cell transfection}

The pGPU6-F-CircEA1-GFP/Neo (Genepharma, Shanghai, China) was constructed as a means to knock down F-circEA1 by targeting the back-splicing junction of F-circEA1, using shNC was used as the negative control. The complete sequence of F-circEA1 was insert into vector pEX3-GCMV-MCS-Neo (Genepharma,Shanghai, China), and an empty vector with no F-circEA1 sequence, was used as the control plasmid. All plasmids were transfected with Lipofectamine 3000 (Life Technologies, USA), and RNA and proteins were collected 48 hours and 72 hours after transfection, respectively. The interference sequence and the full-length sequence of F-circEA1 are presented in Additional file 1. 


\section{RNA or DNA extraction, RNA sequencing and quantification}

Total RNA was extracted from cells using Trizol reagent (Life Technologies, USA) and genomic DNA was extracted from H3122 cells using a genomic DNA mini preparation kit with spin columns (Beyotime, Shanghai, China). To digest the linear RNA, $5 \mu \mathrm{g}$ of total RNA was incubated at $37^{\circ} \mathrm{C}$ for 25 min with 20 units of RNase R (Geneseed, Guangzhou, China) and then reverse transcribed into cDNA with a SMART MMLV Reverse Transcriptase kit (Takara, Dalian, China) according to the manufacturer's instructions. The cDNA was then amplified using Phanta ${ }^{\circledR}$ Max Super-Fidelity DNA Polymerase (Vazyme, Nanjing, China) and the relevant primers by PCR. The products were electrophoresed on an agarose gel and then sequenced in Invitrogen (Shanghai, China). The relative expression levels of the genes were quantified by QRT-PCR using TB Green ${ }^{\circledR}$ Premix Ex Taq ${ }^{\text {TM }}$ (Takara, Dalian, China) and relative expression levels were compared using the $2^{-\Delta \Delta C t}$ method. The primer sequences involved in the above assays are listed in Additional file 1.

\section{Fluorescence in situ hybridization (FISH) of F-circEA1 in H3122 cells}

Cy3 and DAPI were used to label the backsplice junction (BSJ) (Cy3-5区

CAACT+TCATTTGTTGTCA+TGTGTCT-3囚-Cy3) of F-circEA1 and cell nuclei respectively, to observe the position of F-circEA1 in the H3122 cells. FISH technology was performed according to instructions from the RNA FISH kit (Genepharma, Shanghai, China) and images were taken with a fluorescence microscope (Carl Zeiss, Oberkochen, Germany).

\section{Cell proliferation and cell viability assays}

For the cell proliferation assay, cells were seeded in 96-well plates at a concentration of $3 \times 10^{3}$ cells per well and cultured in complete medium (RPMI $1640+10 \% \mathrm{FBS}+1 \%$ penicillin/streptomycin) at $37^{\circ} \mathrm{C}$. Then $10 \mu \mathrm{L}$ of CCK8 was added into each well at fixed time points for 2 hours and the absorbance was measured at $450 \mathrm{~nm}$ using a microplate reader (BioTek, Epoch, USA). For the cell viability assays, $5 \times 10^{3}$ cells per well were seeded and cultured in 96-well plates with complete medium at $37^{\circ} \mathrm{C}$ for 24 hours, then crizotinib(Sigma, Aldrich, USA) or DMSO (as control) (Sigma, Aldrich, USA)were added at different concentrations. After 48 hours, $10 \mu \mathrm{L}$ of CCK 8 was added into each well for 2 hours and the absorbance read at $450 \mathrm{~nm}$ using a microplate reader (BioTek, Epoch, USA).

\section{Transwell migration and invasion assays}

Transwell chambers (Corning, NY, USA) were used for the cell migration and invasion assays. For the invasion assay, the upper chambers were covered with an even layer of Matrigel (BD Biosciences CA, 
USA) diluted with RPMI-1640 (1:6). The cells were suspended in serum-free RPMI-1640 medium and inoculated uniformly into the upper chamber at a volume of $700 \mu \mathrm{L}$ and RPMI-1640 medium containing $10 \%$ FBS was added to the lower chamber. After incubation at $37^{\circ} \mathrm{C}$, cotton wool swabs were used to carefully remove the cells from the upper surface membrane. The cells on the lower surface of the chamber were fixed with methanol for $15 \mathrm{~min}$ and stained with 1\% Crystal Violet (Beyotime, Shanghai, China) for $15 \mathrm{~min}$. At least five different random fields were chosen to image and count the cells under the microscope.

\section{Analysis of the cell cycle and apoptosis by flow cytometry}

Cells were collected and fixed with $70 \%$ ethanol and placed in a refrigerator at $4^{\circ} \mathrm{C}$ for $7-8$ hours, then stained with propidium iodide $(\mathrm{PI})$ solution for half an hour and then the different cell cycle stages were analyzed within one hour by flow cytometry (Mloflox xdp, Beckman, San Jose, CA, USA). To determine cell apoptosis, cells were stained with Annexin V-PE and 7-AAD according to the protocol within the Annexin V-PE/7-AAD apoptosis detection Kit (Vazyme, Nanjing, China) and then the ratio of apoptotic to healthy cells was measured after one hour.

\section{Western blot analysis}

Cell lysates were prepared with RIPA (Beyotime, Shanghai, China) buffer to isolate total cellular protein. The proteins were then resolved by SDS-PAGE using a $10 \%$ gel, and transferred wet to PVDF membranes (Bio-Rad, Hercules, CA, USA). The membranes were blocked with $5 \%$ BSA and the following primary antibody applied overnight at $4^{\circ} \mathrm{C}$ : ALK (D5F3R,1:2,000), p-ALK (1:1,000), PI3K (1:1,000), p-PI3K (1:1,000), AKT $(1: 1,000)$, p-AKT $(1: 2,000)$, mTOR $(1: 1,000)$, p-MTOR $(1: 1,000)$, JAK3 $(1: 1,000)$, p-JAK3 $(1: 1,000)$, STAT3 (1:1,000), p-STAT3 (1:2,000), MEK1/2 (1:1,000), p-MEK1/2 (1:1,000), ERK1/2 (1:1,000), p-ERK1/2 $(1: 1,000)$, GAPDH $(1: 1,000)$ ( all from Cell Signaling Technology, Beverly, MA,USA). The next day, blots were incubated with secondary anti-rabbit (1:3000, Cell Signaling Technology, Beverly, MA,USA) at room temperature for 1 hour. Finally, protein bands were detected with Immobilob ${ }^{\text {TM }}$ Western chemiluminescent HRP substrate (Millipore, Billerica, MA, USA), using a fully automated chemiluminescence imaging analysis system (Tanon 5200, Shanghai, China).

\section{Establishment of stable cell lines and Nude mice xenografts}

Lentiviral interference vector LV3-H1-F-CircEA1-GFP \& Puro, and the control

lentivirus were constructed by Genepharma (Shanghai, China). After infecting H3122 cells, the transfectants were isolated using puromycin selection to form stable cell lines. Next $1 \times 10^{6}$ cells and 30 $\mu \mathrm{L}$ Matrigel (BD Biosciences CA, USA) were diluted with PBS to $100 \mu \mathrm{L}$, and then inoculated into the 
armpit of BALB/c nude mice Subcutaneously (5 weeks old, Nanjing Qinglong Mountain Animal Breeding Farm, China), and maintained in a pathogen-free environment. The body weight and tumor volume of the mice were measured every 3 days after tumor formation. Finally, tumorous tissues were collected after 25 days.

\section{Immunohistochemistry (IHC)}

The transplanted tumor tissue was made into paraffin sections and incubated with anti-ALK (D5F3R 1: 250) primary antibody (Cell Signaling Technology, Beverly, MA, USA) overnight at $4^{\circ} \mathrm{C}$, and incubated with secondary anti-rabbit for one hour at $37^{\circ} \mathrm{C}$, then soaked in HRP-labeled antibody/streptavidin solution for 10 min and stained with diaminobenzidine (DAB). Target protein levels were evaluated based on their intensities within positive cells. Finally, five fields of view were analyzed for each slide, using a fluorescence microscope (Carl Zeiss, Oberkochen, Germany), at a magnification of 400x. The total score was obtained by multiplying the dye intensity and the percentage of the number of cells. The dye intensity score: 0 points (negative), 1 point (light yellow), 2 points (brown yellow), 3 points (dark brown). Percentage of cells: 0 points (negative), 1 point (1-25\% positive cells), 2 points (26-50\% positive cells), 3 points (51-75\% positive cells), 4 points (76-100 \% Positive cells).

\section{Statistical analyses}

Statistical analyses were performed using GraphPad Prism 8.0 and differences between groups were mainly compared using Student's $t$ test. Difference with $p$ value $<0.05$ is statistically significant.The gray value of WB strips value was measured by Image $J$. The dates are reported as the mean \pm standard deviation (SD) by IBM SPSS Statistics 25.

\section{Results}

\section{Specific expression of F-cirEA1 in EML4-ALK1 positive cells.}

We first confirmed the expression of EML4-ALK1 in H3122, A549 and HBE (human bronchial epithelial cell) as negative control cells. PCR products confirmed the presence of the EML4-ALK1 gene in H3122 cells only. We also used RNase R to digest linear RNA and found that linear RNA was able to be degraded by RNase R (Fig. 1a). Using the primers F1/R1 from the PCR products and by sanger sequencing, H3122 cells exhibited the fusion site of EML4-ALK1 (Fig. 1b). Next, the RNA samples were treated with RNase R to remove linear RNA and the divergent primers F2/R2, were used to find the BSJ (the backsplice junction). In order to prevent the interference of genomic DNA, we also used DNA samples of H3122 cells, only one circular RNA was detected and termed F-circEA1 (Fig. 1c). The primer of GAPDH was F3/R3 as control. The PCR product was identified as the binding site of F-cricEA1, which was formed by reverse 
splicing of exons 12-13 of EML4 and exons 20-26 of ALK (Fig. 1d). We then went on to design probes for the BSJ, and using fluorescence in situ hybridization (FISH), we found that F-circEA1 was localized both to the nucleus and cytoplasm in H3122 cells (Fig. 1e).

\section{F-circEA1 contributes to the proliferation, migration and invasion of $\mathrm{H} 3122$ cells.}

To determine a role for F-circEA1 in H3122 cells, we constructed the knockdown plasmid (pGPU6-FCircEA1-GFP/Neo) targeting the backsplice junction of F-circEA1 (Fig. 1d,2a), the interference rate of FcircEA1 was $79.09 \% \pm 3.38 \%$ after transfection of the knockdown plasmid in H3122 cells (Fig. 2b). We also constructed the F-circEA1 overexpression plasmid containing pEX3-GCMV-MCS-Neo vector (Fig. 2c), the overexpression fold of F-circEA1 was $1883.00 \pm 198.23$ after transfection of the overexpression plasmid in H3122 cells (Fig. 2d). CCK8 experiments showed that cell proliferation decreased after knockdown of FcircEA1, but increased after its overexpression (Fig. 2f). Transwell experiments found that F-circEA1 knockdown, attenuated the migration and invasion capability of H3122 cells (fig. $2 \mathrm{~g}$ ) but stimulated them when F-circEA1 was overexpressed (Fig. 2h). These results indicated that F-circEA1 had a pro-oncogenic effect and promoted cancer progression.

\section{F-circEA1 promotes the proliferation, migration and invasion of A549 and SPCA1 cells.}

We also transfected F-circEA1 overexpression plasmid in A549 and SPCA1 cells (no EML4-ALK1 gene). CCK8 suggest that F-circEA1 can promote the proliferation of A549 (Fig. 3a) and SPCA1 (Fig. 3b). Transwell experiments found that F-circEA1 overexpression, promoted the migration and invasion capability of A549 (Fig. 3c) and SPCA1 (Fig. 3d) cells. These results showed that F-circEA1 promotes the proliferation, migration and invasion were not dependent on the existence of the EML4-ALK1 fusion gene.

\section{Knockdown of F-circEA1 induces cell cycle arrest, promotes apoptosis and drug sensitivity to crizotinib in H3122 cells.}

We used flow cytometry to detect the different stages of the cell cycle, and apoptosis in H3122 cells, and found that after knockdown of F-circEA1, the cell synthesis stage of the cell cycle decreased significantly (Fig. 4a), but apoptosis clearly increased (Fig. 4b). These findings indicated further that knockdown of FcircEA1 was not conducive to tumor progression. Crizotinib is a commonly used drug to treat EML4-ALKpositive lung cancer patients. Our CCK8 experimental results showed that $\mathrm{H} 3122$ cell viability decreased significantly with increasing concentration of crizotinib and after these cells were subjected to F-circEA1 knockdown, they showed higher sensitivity to the drug (Fig. 4c). 


\section{F-circEA1 promotes the expression of EML4-ALK1.}

It has been well-established that EML4-ALK1 is an oncogene and that F-circEA1 is derived from EML4ALK1. However, its effects on the expression of EML4-ALK1 is unknown. However, we have detected both mRNA and protein for EML4-ALK1 after knockdown and overexpression of F-circEA1 in H3122 cells. The results of QRT-PCR detection with the primer F4/R4 showed that the mRNA of EML4-ALK1 decreased after knockdown (Fig. 5a), while results were opposite after overexpression of F-circEA1 (Fig. 5b). Next, we used Western blotting to detect ELM4-ALK1 protein in H3122 cells and found that the protein was down-regulated after F-circEA1 knockdown (Fig. 5c), but up-regulated when F-circEA1 was overexpressed (Fig. 5d). These findings suggest that F-circEA1 may affect the biological function of the H3122 cells by interfering with the expression of EML4-ALK1.

\section{F-circEA1 activates the downstream signaling pathway of ALK.}

It has been reported that the downstream signaling pathways related to EML4-ALK include principally PI3K-AKT-mTOR, JAK3-STAT3, and MEK-ERK1/2 [19]. The mRNA expression of the above signaling factors were not significantly different after both knockdown or overexpression of F-circEA1 by QRT-PCR (Fig. 6a,6b). Interestingly, we found that the protein expression of these factors was down-regulated after knockdown of F-circEA1 but were significantly up-regulated after F-circEA1 overexpression (Fig. 6c, 6d). It is widely known that these pathways have clear effects on the malignant behavior of tumors, in particular their cell survival, proliferation, and metastasis. Our experimental results showed that F-circEA1 promoted the expression of the parental gene EML4-ALK1, which then activated the downstream signaling pathways related to the parental gene, thereby participating in the malignant biological behavior of lung cancer.

\section{Knockdown of F-circEA1 inhibits subcutaneous xenograft growth in nude mice and the expression of EML4-ALK1 protein in their tumors.}

In vivo, the shF-circEA1 plasmid was inserted into LV3 to form a lentiviral knockdown plasmid and its transfection diagram in H3122 cells (Fig. 7a). These subcutaneous tumor formation experiments in nude mice, showed that knockdown of F-circEA1 had no obvious detrimental effect on body weight (Fig. 7b) but did significantly inhibited the growth of the subcutaneous xenografts, including volume and weight (Fig. 7c, 7d). IHC detected EML4-ALK1 protein as being present in the tumors but this was significantly inhibited after knockdown of F-circEA1 (Fig. 7e). This is consistent with our cell experiments, which showed that F-circEA1 affected the expression of EML4-ALK1 and participated in tumor proliferation. 


\section{Discussion}

CircRNA is different from linear RNA, as both the $5^{\prime}$ cap and $3^{\prime}$ tails are absent, instead it forms a covalently closed loop structure with no 5'-3' polarity, or polyadenylation tail. Therefore, circRNA is resistant to ribonuclease $\mathrm{R}$ treatment $[7,8]$. Recently, it has been found that circRNAs play an important role in the pathogenesis of cancer and can affect many characteristics of the disease. In addition, circRNA can be found in body fluids (Such as blood and saliva) with non-invasion, therefore circular RNA has great potential as a cancer biomarker [9-13].

Chromosome translocation is one important carcinogenic driving mechanism in cancer and different types of chromosomal translocations lead to the expression of specific F-circRNAs in related tumor cells [5]. F-circRNA-hC, F-circRNA-hD, F-circRNA-hE, and F-circRNA-hF have all been found in NPM1-ALKpositive fusion gene tumors [14]. F-circPR can be found in PML-RARa-positive acute promyelocytic leukemia and F-circM9 expression is present in MLL-AF9-positive acute myeloid leukemia. The expression of F-circPR and F-circM9 results in increased cell proliferation and transformation [5].

Our experiments have revealed that there was a fusion circular RNA present in EML4-ALK1 positive lung cancer cells. Using sequencing of PCR products, we have confirmed that F-circEA1 is formed by the reverse connection exons 12-13 of EML4, with exon 20-26 of ALK. Furthermore, research has also found that F-circEA1 promoted tumor cell proliferation, migration, and invasion, these functions were not dependent on the existence of the EML4-ALK1 fusion gene, and was also involved in the regulation of the cell cycle and apoptosis.

Treatment with crizotinib an inhibitor of ALK phosphorylation promoted drug sensitivity in $\mathrm{H} 3122$ cells after interference with F-circEA1, revealing that F-circEA1 is resistant to crizotinib. In vivo, studies have shown that the growth rate of xenogeneic tumors declined and the protein expression level of EML4-ALK1 was significantly decreased in transplanted tumors after this interference by F-circEA1. These results confirmed a role for F-circEA1 in carcinogenesis. To date many studies have found that ALK fusion genes have obvious carcinogenic potential because the abnormal tyrosine kinase activity enhances cell proliferation and survival, and leads to cytoskeletal rearrangement and morphological changes [15 -18]. Our study has confirmed that F-circEA1 positively regulated EML4-ALK1 regardless of mRNA or protein.

Many studies have demonstrated that the downstream signaling pathways affected by EML4-ALK fusion protein are as follows: the RAS-RAF-MEK-ERK pathway which regulates cell proliferation and the PI3KAKT-mTOR and the JAK3-STAT3 pathways involved in cell survival [19]. In our experiments F-circEA1 promoted protein expression of the signal factors involved in the downstream pathway of ALK. These finding supported a role for F-circEA1 in the activation of the downstream signaling of ALK, by promoting the expression of EML4-ALK1 and may further influence the biological function of tumor cells. However, the mechanism of F-circEA1 affecting EML4-ALK1 needs to be further explored. In our experiments, FcircEA1 promoted the protein expression of the main signal molecules involved in the downstream pathway of ALK. But it did not affect the mRNA expression of these signal molecules. It is considered that their expression may be affected at the post-transcriptional or translational level, or by regulating the 
expression of EML4-ALK1, which in turn affects the expression of ALK downstream signal proteins, which needs to be further clarified.

\section{Conclusion}

Our research has confirmed a direct effect of F-circEA1 on the expression of the parental gene EML4ALK1, and may activate its downstream signaling pathways. We have also found for the first time, a direct effect of circRNA on the downstream signaling pathways of three of the parental genes and this can further affect the biological function of tumor cells. We have also provided a new role for fusion circRNAs in cancers and that F-circEA1 has potential drug resistance to crizotinib, resulting in a novel treatment for EML4-ALK variant 1 positive NSCLC. However, the mechanism of F-circEA1 affecting EML4ALK1 needs to be further clarified.

\section{Abbreviations}

EML4-ALK1, echinoderm microtubule associated-protein like 4-anaplastic lymphoma kinase variant 1; NSCLC, non-small cell lung cancer; NPM1-ALK, nucleophosmin 1-anaplastic lymphoma kinase; PMLRARa, promyelocytic leukemia-retinoic acid receptor a; SLC34A2-ROS1, solute carrier family 34 member A2- reactive oxygen species proto-oncogene 1; BSJ, the backsplice junction; IHC, immunohistochemistry; $\mathrm{FISH}$, fluorescence in situ hybridization.

\section{Declarations}

\section{Ethics approval and consent to participate}

All institutional and national guidelines for the care and use of laboratory animals were followed. All animal experiments were approved by the Institutional Ethics Committee of Jinling Hospital, China (2020JLHGKJDWLS-39).

\section{Consent for publication}

Not applicable.

\section{Availability of data and materials}

The data supporting the conclusions of this article is included within the article and its additional file.

\section{Funding}


The study was supported by the National Natural Science Foundation of China Youth Fund (Grant Nos. 81572273), the key Young Medical Talents in Jiangsu Province (No. QNRC2016911), and the Nanjing Medical Science and Technology Development Project (No. YKK16246).

\section{Competing interests}

The authors have no conflicts of interest to declare.

\section{Authors' Contributions}

$\mathrm{YH}, \mathrm{TL}, \mathrm{HL}$ and $\mathrm{YS}$ participated in the design of the study. $\mathrm{HL}$ and $\mathrm{YS}$ helped to revise the manuscript. YH carried out the experiment and drafted the manuscript. HL, YS, YH, TL, MY, SZ and JL participated in the date analysis and the statistical analysis. All authors read and approved the final manuscript.

\section{Acknowledgments}

Not applicable.

\section{References}

1. Pawlak K., Gabryel P., Kujawska A., Kasprzyk M., Piwkowski C., Kuffel B., et al. Long-term results of surgical treatment of non-small cell lung cancer in patients over 75 years of age. Kardiochir Torakochirurgia Pol. 2018;15(2):65-71. https://doi: 10.5114/kitp.2018.76470.

2. Herbst R.S., Morgensztern D., Boshoff C. The biology and management of non-small cell lung cancer. Nature. 2018;553(7689):446-454. https://doi:10.1038/nature25183

3. Sullivan H.C., Fisher K.E., Hoffa A.L., Wang, J., Saxe, D., Siddiqui, M.T., et al. The Role of Immunohistochemical Analysis in the Evaluation of EML4-ALK Gene Rearrangement in Lung Cancer. Appl Immunohistochem Mol Morphol. 2015;23(4):239-244.

https://doi:10.1097/PAl.0000000000000088

4. Butrynski J.E., D'Adamo D.R., Hornick J.L., Cin P.D., Shapiro G.I. Crizotinib in ALK-rearranged inflammatory myofibroblastic tumor. N Engl J Med. 2010;363(18):1727-33. https://doi:10.1056/NEJMoa1007056

5. Guarnerio J., Bezzi M., Jeong J.C., Paffenholz S., Pandolfi P. Oncogenic Role of Fusion- circRNAs Derived from Cancer-Associated Chromosomal Translocations. Cell. 2016;166(4):1055-56. https://doi:10.1016/j.cell.2016.07.035

6. Wu K., Liao X., Gong Y., Zhou J.K., Tan S., Pu,W.,Huang C., Wei Y.Q., Peng Y. Circular RNA F-circSR derived from SLC34A2-ROS1 fusion gene promotes cell migration in non-small cell lung cancer. Mol Cancer. 2019;18(1):98. https://doi:10.1186/s12943-019-1028-9 
7. Chen L.L., Yang L. Regulation of circRNA biogenesis. RNA Biol. 2015;12(4):381-388. https://doi:10.1080/15476286.2015.1020271

8. Suzuki H., Tsukahara T. A view of pre-mRNA splicing from RNase R resistant RNAs. Int J Mol Sci. 2014;15(6):9331-42. https://doi:10.3390/ijms15069331

9. Hanahan D., Weinberg R.A. Hallmarks of cancer: the next generation. Cell. 2011;144(5): 646-674. https://doi:10.1016/j.cell.2011.02.013

10. Bahn J.H., Zhang Q., Li F., Chan T.M., Lin X., Kim Y. Wong David T.W., et al. The landscape of microRNA, Piwi-interacting RNA, and circular RNA in human saliva. Clin Chem. 2015; 61(1):221-230. https://doi:10.1373/clinchem.2014.230433

11. Li Y., Zheng Q., Bao C., Li S., Guo W., Zhao J., et al. Circular RNA is enriched and stable in exosomes: a promising biomarker for cancer diagnosis. Cell Res. 2015;25(8):981-984. https:// doi:10.1038/cr.2015.82

12. Wang F., Nazarali A. J., Ji S. Circular RNAs as potential biomarkers for cancer diagnosis and therapy. Am J Cancer Res. 2016; 6(6): 1167-76.

13. Kim KM, Abdelmohsen $K$, Mustapic M, Kapogiannis D, Gorospe M. RNA in extracellular vesicles. Wiley Interdiscip Rev RNA. 2017; 8(4): e1413. https://doi:10.1002/wrna.1413

14. Loelia, Babin, Marion, Piganeau, Benjamin, Renouf, et al. Chromosomal Translocation Formation Is Sufficient to Produce Fusion Circular RNAs Specific to Patient Tumor Cells. Iscience. 2018; 5:1929.https://doi:10.1016/j.isci.2018.06.007

15. Kuefer M.U., Look A.T., Pulford K., Behm F. G, Morris S.W. Retrovirus -mediated gene transfer of NPM-ALK causes lymphoid malignancy in mice. Blood. 1997;90(8):2901-2910. https://doi:10.1016/S0268-9499(95)80077-8

16. Chiarle R., Gong J. Z., Guasparri I., Pesci A., Inghirami G. NPM-ALK transgenic mice spontaneously develop T-cell lymphomas and plasma cell tumors. Blood. 2003;101(5):1919-27. https://doi:10.1182/blood-2002-05-1343

17. Wellmann A., Doseeva V., Butscher W., Raffeld M., Fukushima P., Stetlerstevenson M., et al. The activated anaplastic lymphoma kinase increases cellular proliferation and oncogene up-regulation in rat 1a fibroblasts. Faseb J. 1997;11(12):965-72. https://doi:10.1096/fasebj.11.12.9337149

18. Ambrogio C., Voena C., Manazza A. D., Piva R., Chiarle R. p130Cas mediates the transforming properties of the anaplastic lymphoma kinase. Blood. 2005;106(12):390716.https://doi:10.1182/blood-2005-03-1204

19. Shaw A.T., Solomon B. Targeting Anaplastic Lymphoma Kinase in Lung Cancer[J]. Clin Cancer Res. 2011;17(8):2081-6. https://doi:10.1158/1078-0432

\section{Figures}


Fig. 1

a

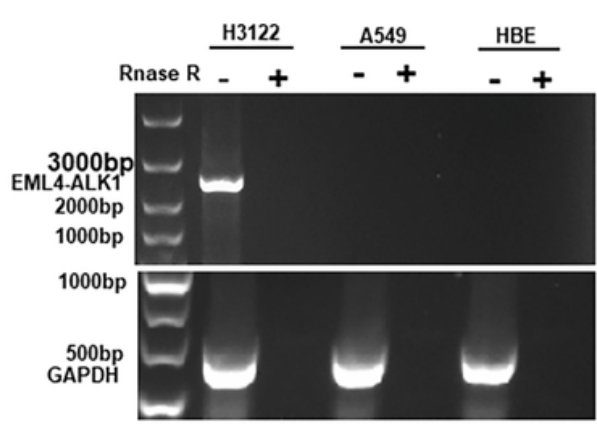

b

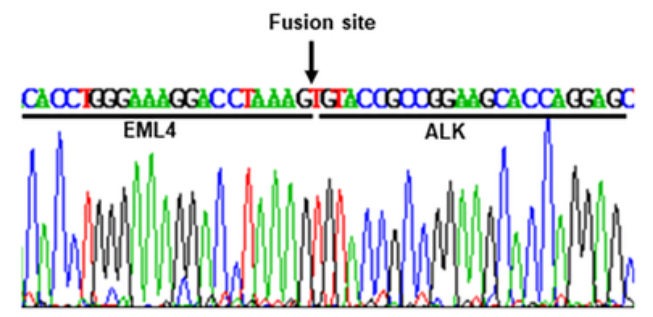

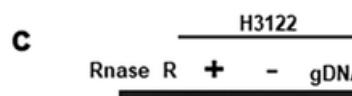

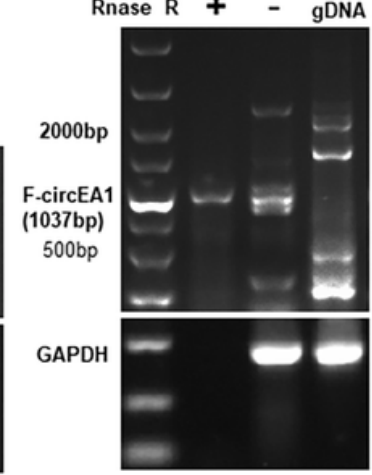

e d

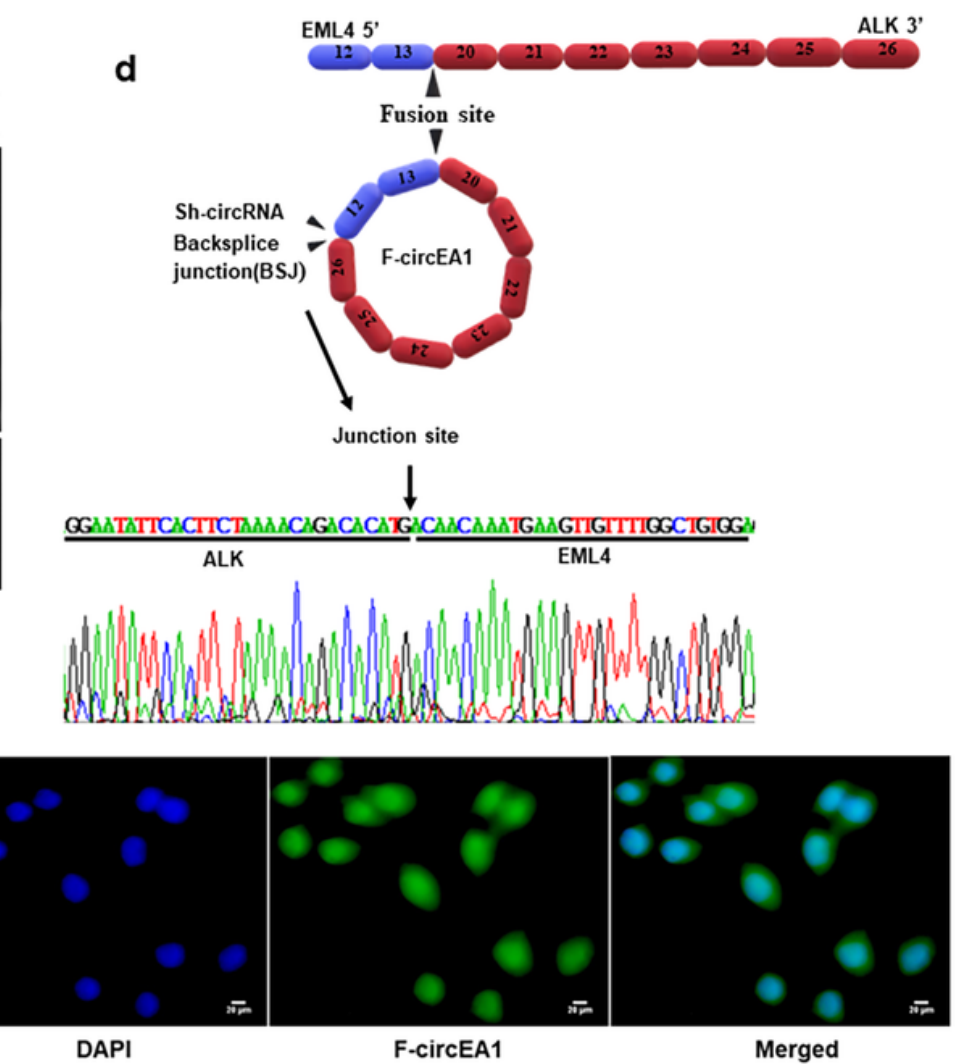

\section{Figure 1}

Identification of F-circEA1. a EML4-ALK1 and GAPDH were amplified from the CDNA of the cells, the RNAs of the cells were treated with and without RNase R, respectively. $b$ The PCR product was identified as the fusion site of EML4-ALK1. c F-circEA1 and GAPDH were detected and amplified from the PCR products of the RNase R-treated, without the RNase R-treated, and the genomic DNA, respectively. $d$ The PCR product was identified as the junction site of F-cricEA1. e F-circEA1 was localized both to the nucleus and cytoplasm in H3122 cells, DAPI was used to label the cell nuclei. (Scale bar=20 $\mu \mathrm{m}$ ). 
Fig. 2

a

a
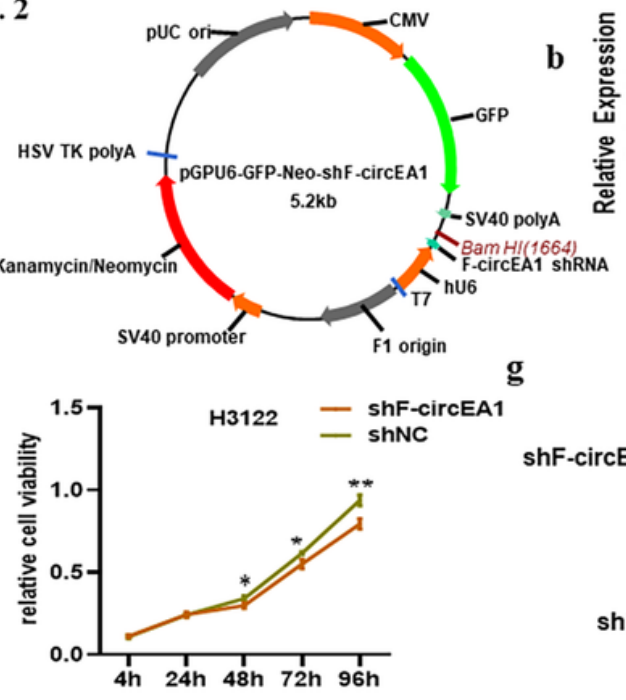

f

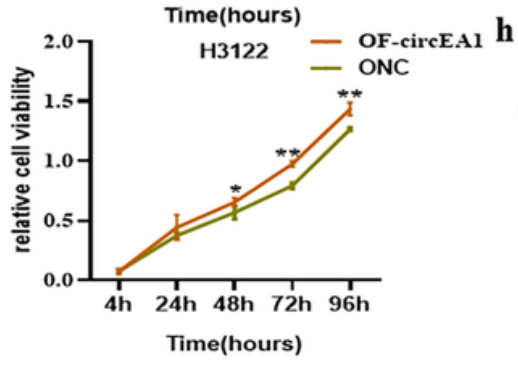

g
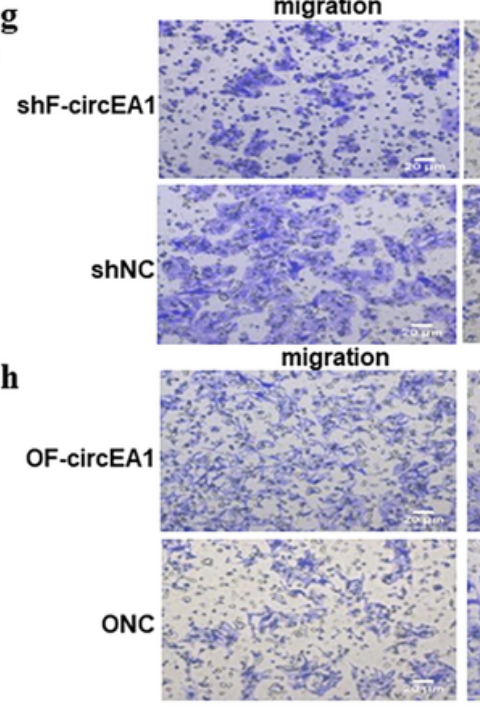

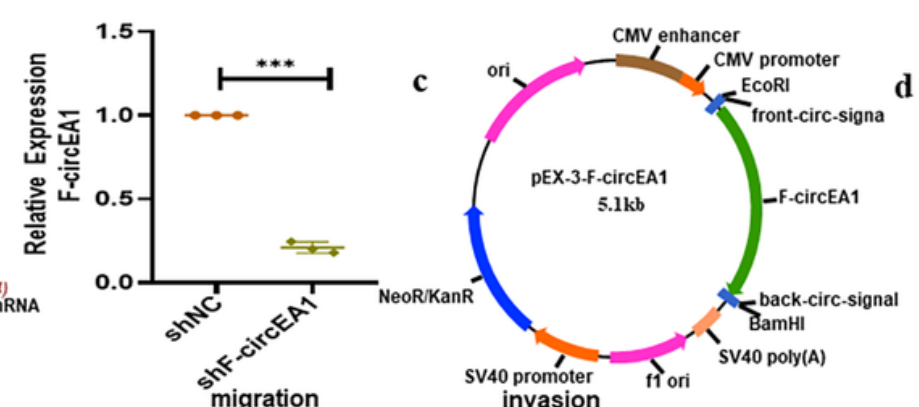

invasion

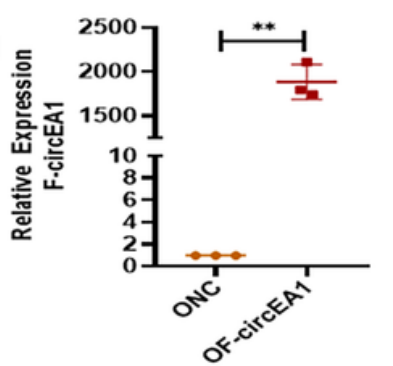

H3122

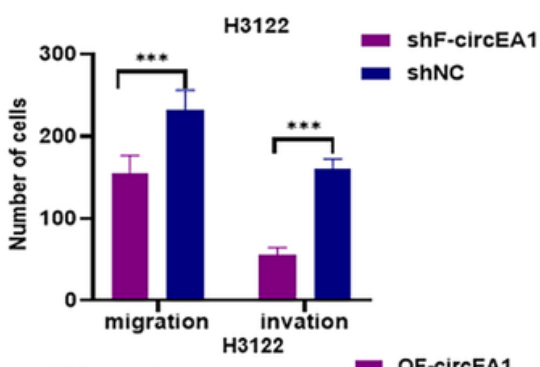

invasion
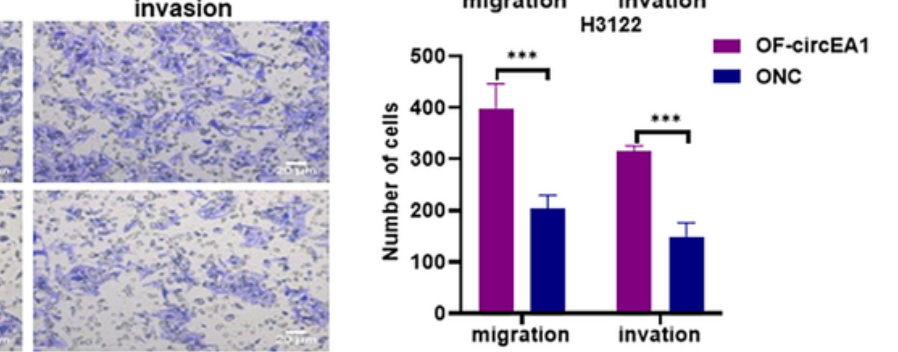

Figure 2

F-circEA1 promotes the proliferation, migration and invasion of $\mathrm{H} 3122$ cells. a The diagram of the knockdown plasmid(pGPU6-GFP-Neo-F-CircEA1). b The expression of F-circEA1 after transfection of the knockdown plasmid. $c$ The diagram of pEX-3-GCMV-F-circEA1-Neo-expression plasmid. $d$ The expression of F-circEA1 after transfection of the overexpression plasmid. e The proliferation of H3122 cells after knockdown and non-knockdown of F-circEA1. $f$ The proliferation of H3122 cells with and without FcircEA1 overexpression. $g$ Images and quantification of the migration and invasion with F-circEA1 interference in $\mathrm{H} 3122$ cells. $\mathrm{h}$ Images and quantification of the migration and invasion with F-circEA1 overexpression in $\mathrm{H} 3122$ cells. (Scale bar $=20 \mu \mathrm{m})$. The data statistics are mean $\pm S D,\left({ }^{*} P<0.05, * \star P<0.01\right.$, $\star \star \star P<0.005)$. 
Fig. 3

a

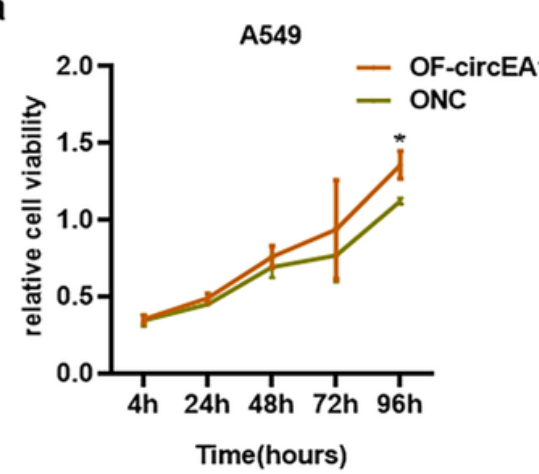

b

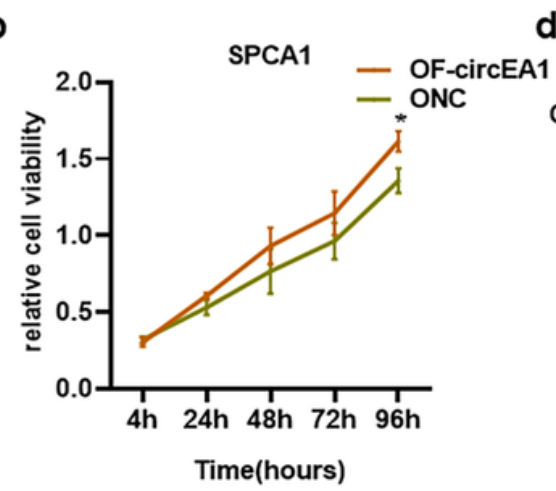

c

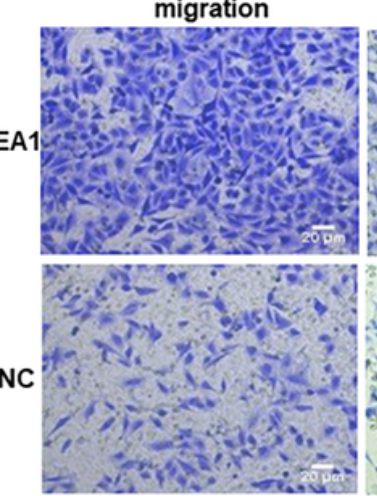

migration
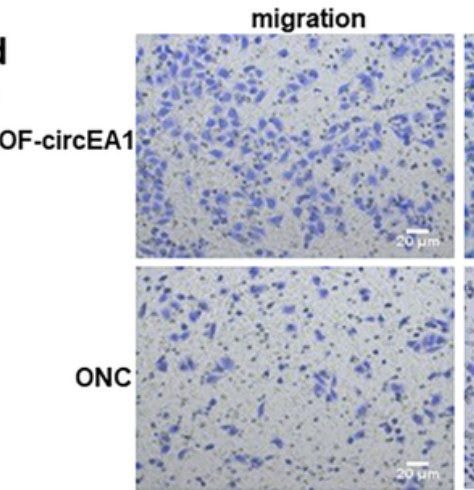

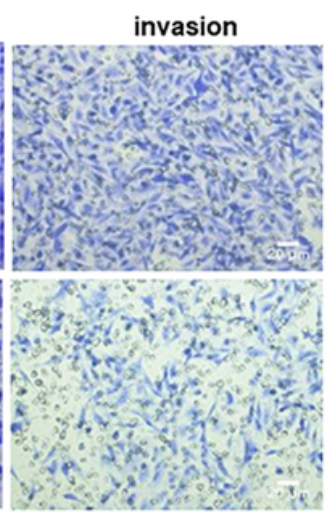

invasion

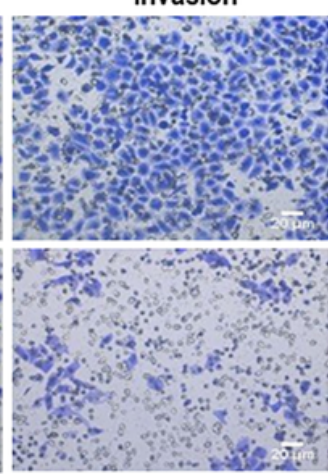

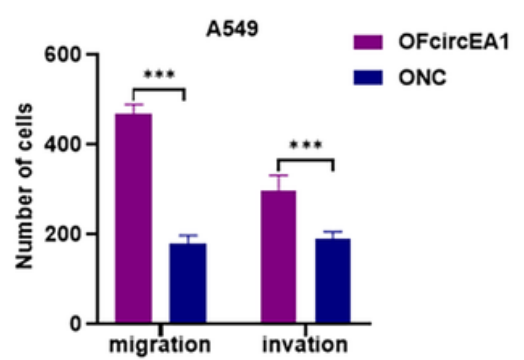

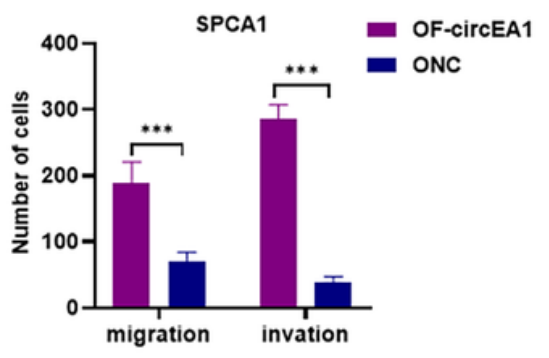

Figure 3

F-circEA1 promotes the proliferation, migration and invasion of A549 and SPCA1 cells. a-b The proliferation of A549 and SPCA1cells with F-circEA1 overexpression. c-d Images and quantification of the migration and invasion with F-circEA1 overexpression in A549 and SPCA1 cells. (Scale bar=20 $\mu \mathrm{m})$. The data statistics are mean $\pm S D,(* P<0.05, * * P<0.01, * * * P<0.005)$. 
Fig. 4

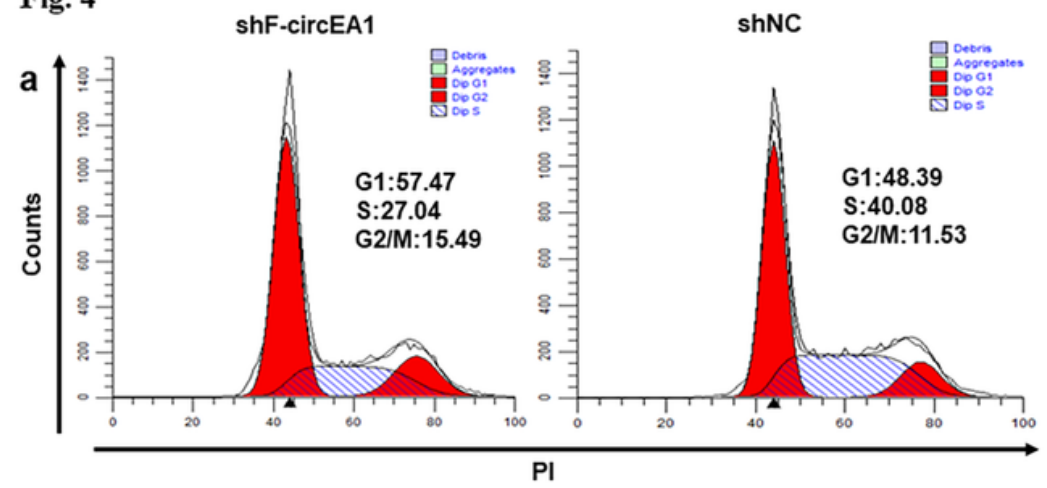

b

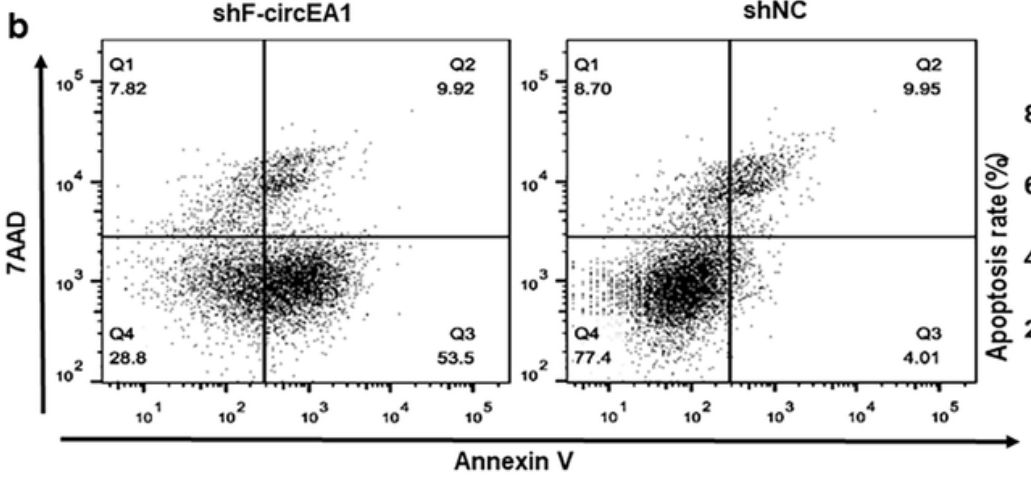

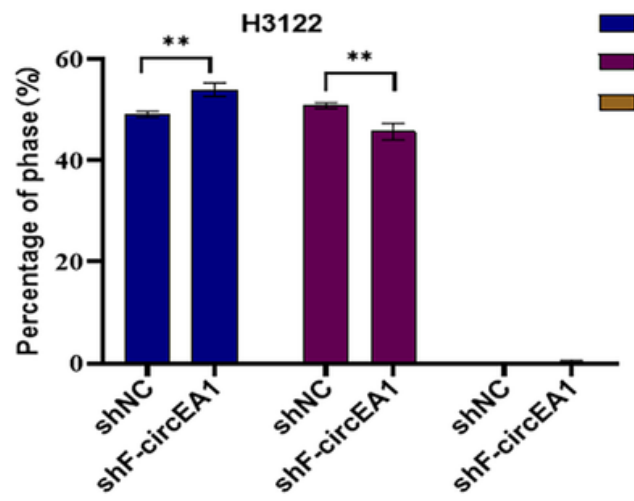

c
G1

E

G2/M

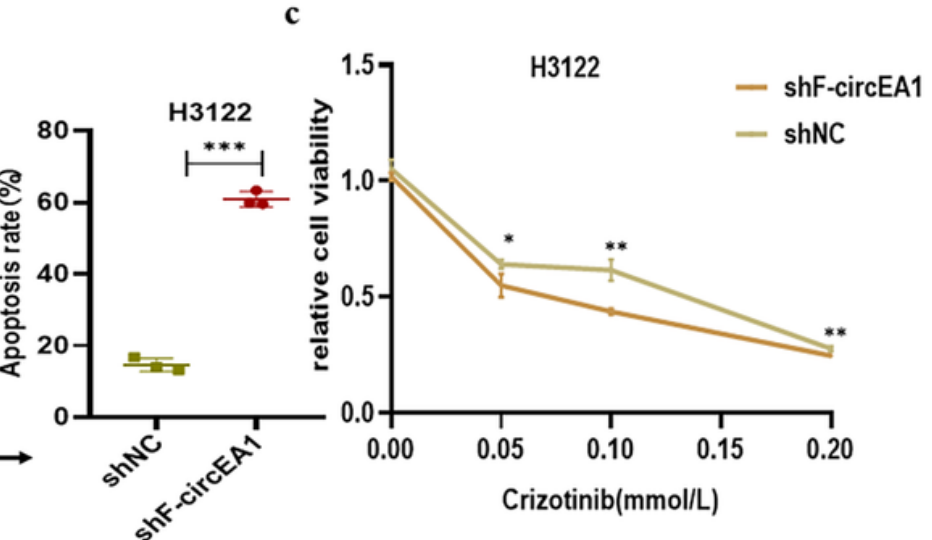

Figure 4

Knockdown of F-circEA1 induces cell cycle arrest, promotes apoptosis and improves the sensitivity to crizotinib. a-b The different stages of the cell cycle and the apoptosis rate were quantified of H3122 cells after knockdown of F-circEA1. c Knockdown of F-circEA1 H3122 cell viability was assessed with different concentration of crizotinib after these cells were subjected to F-circEA1 knockdown. The data statistics are mean $\pm S D,\left({ }^{\star} P<0.05,{ }^{*} \mathrm{P}<0.01,{ }^{*} * * \mathrm{P}<0.005\right)$. 
Fig. 5

a

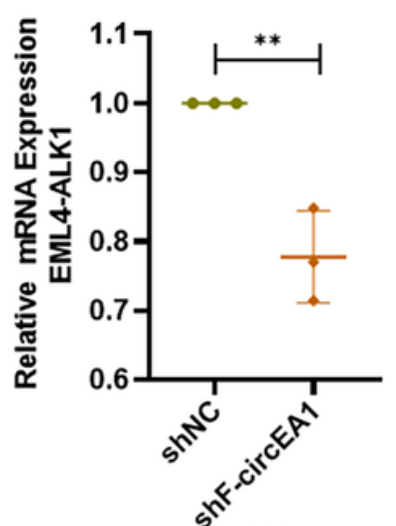

b

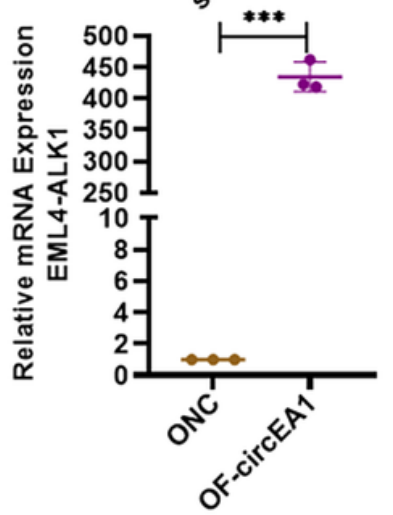

C
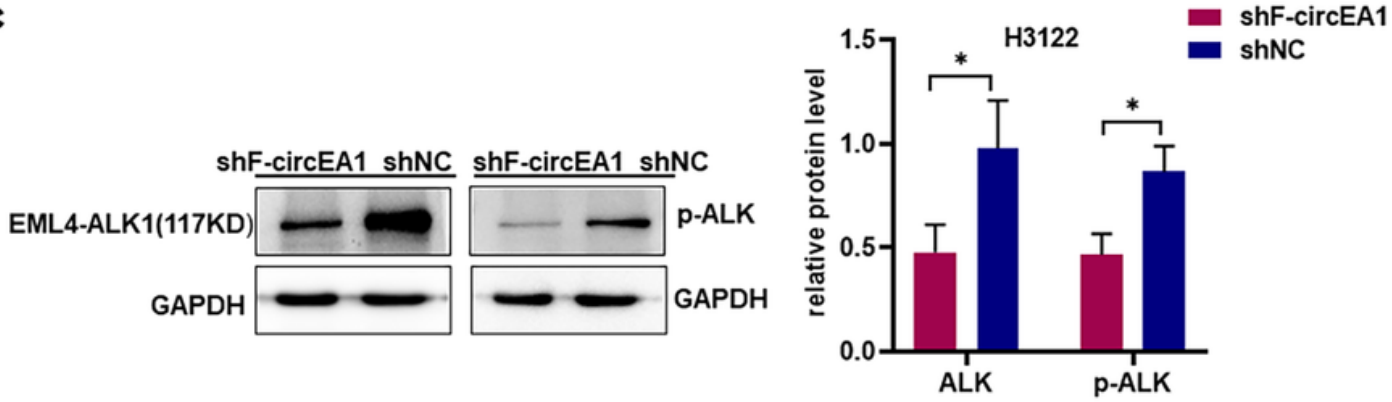

d
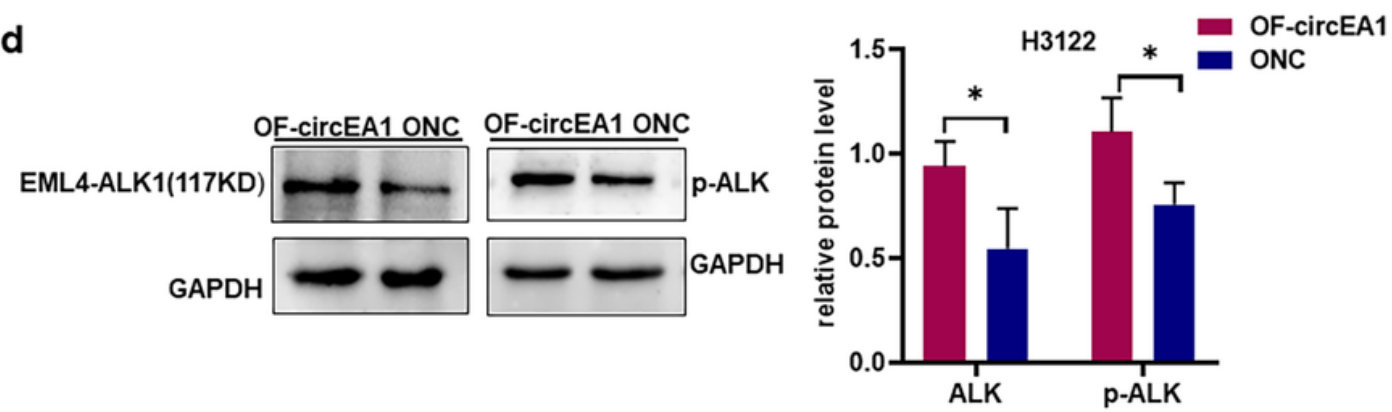

Figure 5

F-circEA1 promotes the expression of EML4-ALK1 in H3122 cells. a-b The mRNA expression of EML4ALK1 were measured after F-circEA1 knockdown and overexpression. c-d The protein of EML4-ALK1 were detected after F-circEA1 knockdown and overexpression. The dates of QRT-PCR are compared with 2- $\Delta \Delta$ Ct method. The data statistics are mean $\pm S D,\left({ }^{*} P<0.05,{ }^{*} P<0.01,{ }^{*} * * P<0.005\right)$. 
Fig. 6

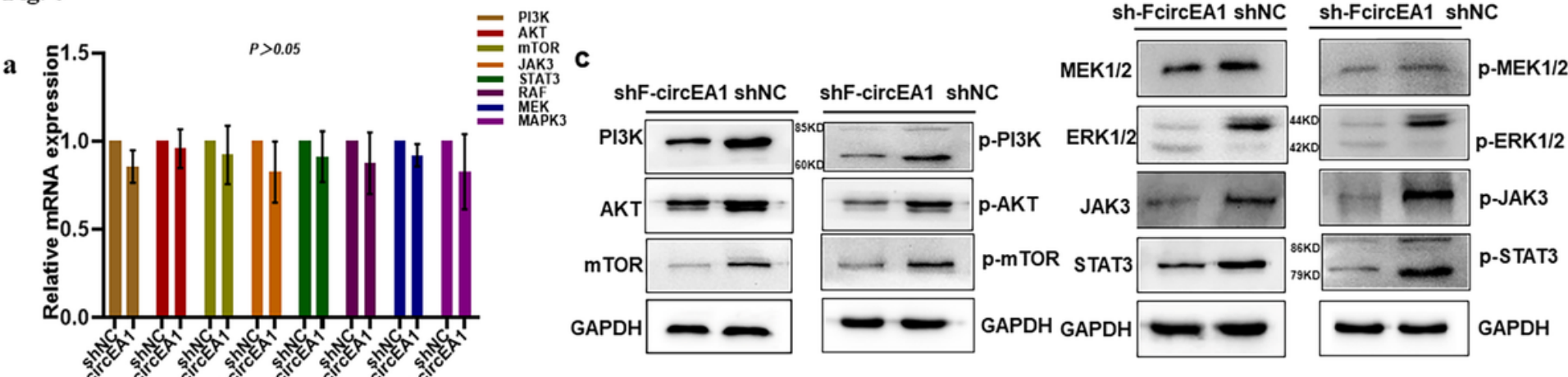

b

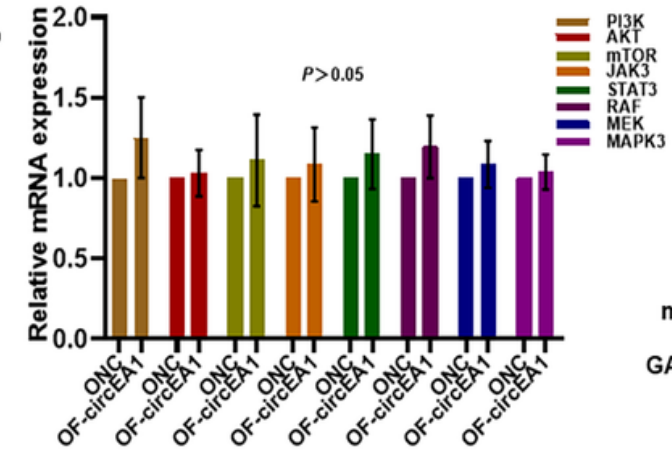

\section{Figure 6}

F-circEA1 activates EML4-ALK1 associated downstream signaling pathways in H3122 cell. a-b The mRNA expression of the signaling factors were determined after transfection of F-circEA1 interference and expression plasmid. c-d The protein expression of the signal factors were detected after F-circEA1 knockdown and overexpression. The dates of QRT-PCR are compared with 2- $\Delta \Delta$ Ct method. The data statistics are mean $\pm S D,\left({ }^{*} P<0.05,{ }^{*} P<0.01, * * * P<0.005\right)$.

Fig. 7

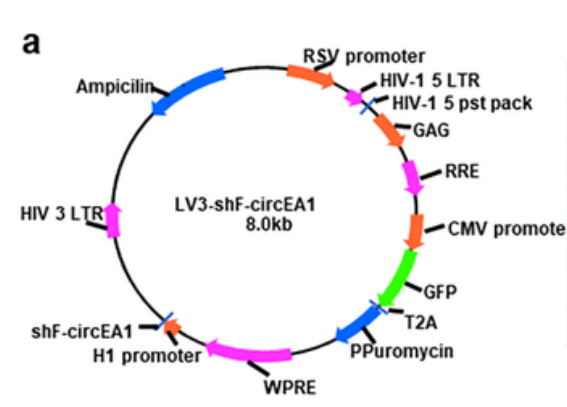

d

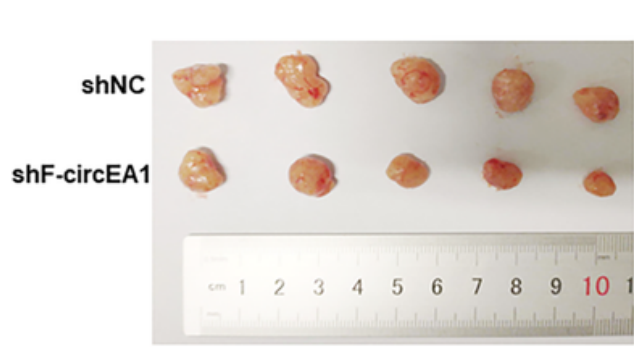

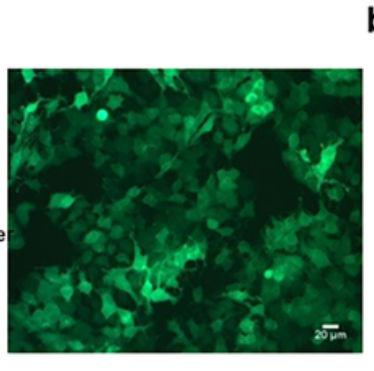
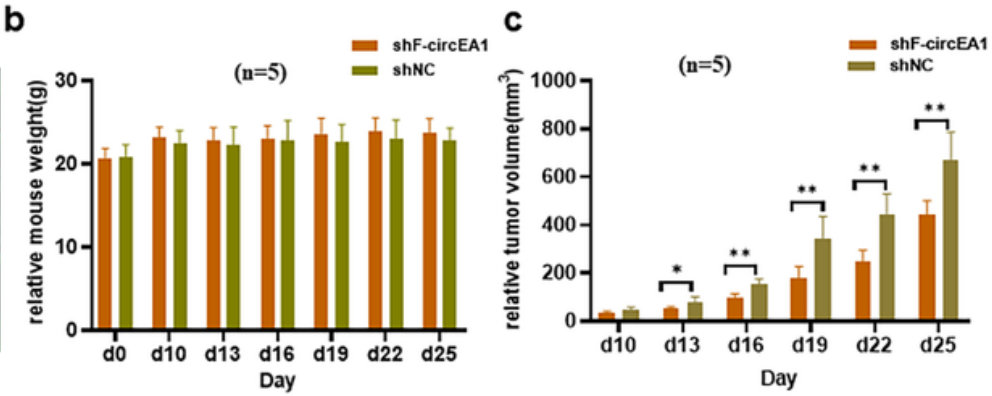

e
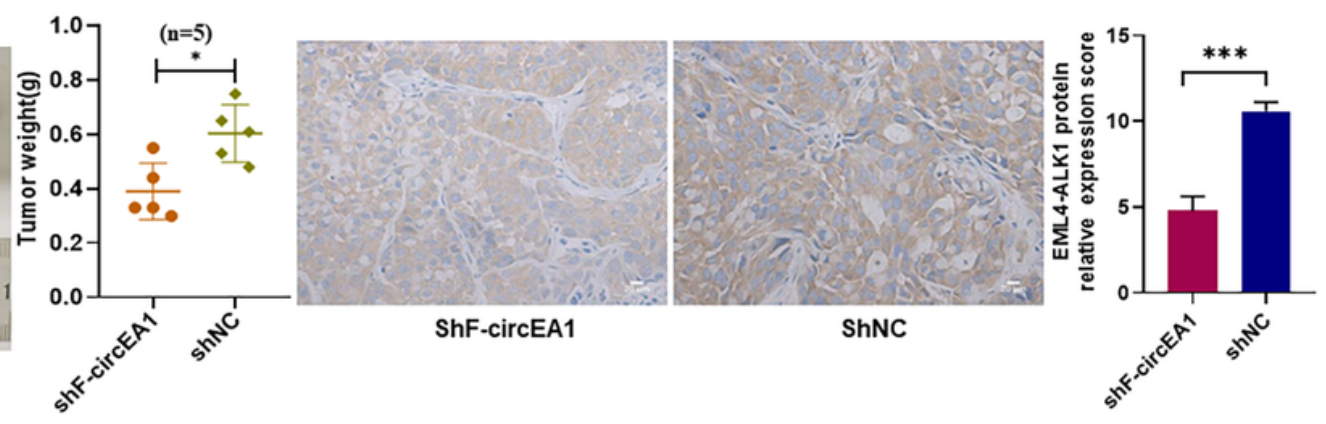


\section{Figure 7}

Knockdown of F-circEA1 inhibited subcutaneous xenograft growth and the protein of EML4-ALK1 in their tumors. a Schematic diagram of lentivirus LV3-H1-shF-CircEA1-GFP\&Puro and its transfection diagram in H3122 cells. b The body weight of the nude mice were no obvious diffence after knockdown of F-circEA1. $c-d$ The tumor volume and weight were measured each three days after knockdown of F-circEA1. e The protein EML4-ALK1 expressed on the cytoplasm and cell membrane of H3122 cells decreased in the group of shF-circEA1 of transplanted tumors. (Scale bar $=20 \mu \mathrm{m}$ ). The data statistics are mean $\pm S D$, $\left({ }^{\star} \mathrm{P}<0.05,{ }^{\star *} \mathrm{P}<0.01,{ }^{* \star *} \mathrm{P}<0.005\right)$.

\section{Supplementary Files}

This is a list of supplementary files associated with this preprint. Click to download.

- Additionalfile1.docx 